\title{
Application of cationic polymer prepared from sulfuric acid lignin as a retention aid for usual rosin sizes to neutral papermaking
}

\begin{abstract}
Cationic polymers that acted as the retention aids for usual rosin sizes in neutral papermaking were prepared from sulfuric acid lignin (SAL), one type of acid lignin. To convert SAL to the cationic polymer (MP-SAL), SAL was phenolated and then treated by the Mannich reaction to introduce the amino groups. In the MP-SAL single system, MP-SAL exhibited high sizing effectiveness in neutral papermaking with the rosin emulsion size. However, MP-SAL showed no sizing effectiveness when soap rosin size was used. MP-SAL showed increased sizing effectiveness as the $\mathrm{p} K_{\mathrm{a}}$ of the introduced amino group increased. From this and comparison of the sizing degrees of MP-SAL and polyethylenimine, which possesses a linear structure, it was suggested that the sizing effectiveness was not only affected by the charge density and molecular weight, but also by the basicity of the introduced amino groups and the molecular structure of the retention aid. In the alum-MP-SAL dual system, alum and MP-SAL synergistically enhanced the sizing effectiveness in the rosin emulsion sizing at neutral $\mathrm{pH}$. In turn, this allowed a decrease in the MP-SAL dosage and resulted in a small decrease in brightness.
\end{abstract}

Key words Lignin $\cdot$ Rosin size $\cdot$ Retention $\cdot$ Mannich reaction $\cdot$ Paper

Y. Matsushita $(\bowtie) \cdot$ A. Iwatsuki ${ }^{1} \cdot S$. Yasuda

Division of Biological Material Sciences, Department of Biosphere

Resources Science, Graduate School of Bioagricultural Sciences,

Nagoya University, Furo-cho, Chikusa-ku, Nagoya 464-8601, Japan

Tel. +81-52-789-4174; Fax +81-52-789-4163

e-mail: ysmatsu@agr.nagoya-u.ac.jp

Present address:

${ }^{1}$ Japan Food Research Laboratories, Nagoya 460-0011, Japan

Part of this report was presented at the 48th Lignin Symposium, October 2003, Fukui

\section{Introduction}

Much attention has been paid to the utilization of biomass energy as a potential replacement for fossil fuel energy because of its abundance and renewable nature. Among procedures using biomass, the acid saccharification of woody materials is an effective method for the production of monosaccharides and ethanol. However, this method yields a large amount of less reactive acid lignin as a byproduct. One of the key considerations in developing this acid saccharification process is to find effective ways to utilize acid lignin.

Acid lignin possesses a highly condensed structure, which is derived from intermolecular dehydrative condensation $^{1,2}$ between benzylic carbons and aromatic nuclei. Recently, the condensed aromatic nuclei of sulfuric acid lignin (SAL), a type of acid lignin with a diarylmethane structure, were selectively exchanged with phenol in the presence of a sulfuric acid catalyst. ${ }^{3}$ As a result, the reactivity of phenolized sulfuric acid lignin (P-SAL) is enhanced because a reactive $p$-hydroxyphenyl moiety is introduced at the side chain $\alpha$-position instead of condensed-type aromatic nuclei. Thus, P-SAL could be easily converted to functional materials by chemical reactions. ${ }^{4-8}$

Incidentally, papermaking conditions have shifted from acidic to neutral-alkaline in recent years because of the closed-circuit papermaking system, the use of recycled waste paper, and lower cost. In our previous study, cationic polymers (MP-SAL), which have high surface activity, were prepared from P-SAL by the Mannich reaction ${ }^{7}$ (Fig. 1) and it was found that they could serve as a retention aid for the rosin-ester emulsion size in neutral papermaking. ${ }^{9}$

Usual rosin sizes such as rosin emulsion size and soap rosin size function under acidic conditions as size reagents, and as rosin-ester emulsion sizes under neutral or alkali conditions. The rosin-ester emulsion size, however, needs about three times the addition than that of synthesized size reagents such as alkylketen dimers and alkenylsuccinic anhydrides. $^{10}$ 
Table 1. Mannich reaction conditions of phenolized sulfuric acid lignin and properties of the products

\begin{tabular}{|c|c|c|c|c|c|c|c|}
\hline \multirow[t]{3}{*}{ MP-SAL } & \multicolumn{4}{|c|}{ Reaction conditions $\mathrm{s}^{\mathrm{a}}$} & \multicolumn{2}{|c|}{ Reaction products } & \multirow{3}{*}{$\begin{array}{l}\text { Sizing degree }{ }^{\mathrm{b}} \text { of } \\
\text { handsheet }(\mathrm{s})\end{array}$} \\
\hline & \multirow[t]{2}{*}{ Amine } & \multicolumn{2}{|l|}{ Dosage (\%) } & \multirow[t]{2}{*}{ Time (h) } & \multirow[t]{2}{*}{ Yield (\%) } & \multirow[t]{2}{*}{$\mathrm{N}$ content $(\%)$} & \\
\hline & & Amine (mmol) & $\mathrm{HCHO}(\mathrm{mmol})$ & & & & \\
\hline 1 & $\mathrm{HN}\left(\mathrm{CH}_{3}\right)_{2}$ & 0.16 & 0.11 & 4 & 78 & 4.2 & 38 \\
\hline 2 & $\mathrm{HN}\left(\mathrm{CH}_{3}\right)_{2}$ & 0.16 & 0.11 & 6 & 88 & 4.3 & 39 \\
\hline 3 & $\mathrm{HN}\left(\mathrm{CH}_{3}\right)_{2}$ & 1.6 & 1.1 & 24 & 90 & 4.4 & 43 \\
\hline 4 & $\mathrm{HN}\left(\mathrm{C}_{2} \mathrm{H}_{5}\right)_{2}$ & 2.6 & 1.1 & 24 & 78 & 3.8 & 47 \\
\hline 5 & $\mathrm{H}_{2} \mathrm{NCH}_{3}$ & 1.1 & 1.1 & 72 & 78 & 4.2 & 54 \\
\hline 6 & $\mathrm{H}_{2} \mathrm{NC}_{2} \mathrm{H}_{5}$ & 1.6 & 1.1 & 72 & 89 & 3.8 & 58 \\
\hline
\end{tabular}

MP-SAL, cationic polymer prepared from sulfuric acid lignin

${ }^{a}$ Phenolized sulfuric acid lignin $(100 \mathrm{mg})$ was reacted at $60^{\circ} \mathrm{C}$ in the mixed solution of $80 \%$ aqueous dioxane $(10 \mathrm{ml})$ and $\mathrm{AcOH}(4 \mathrm{ml})$

${ }^{\mathrm{b}}$ Sizing degree of handsheet prepared with the product $(1 \%)$ and rosin emulsion size $(0.35 \%)$ in MP-SAL single system at $\mathrm{pH} 7.5$

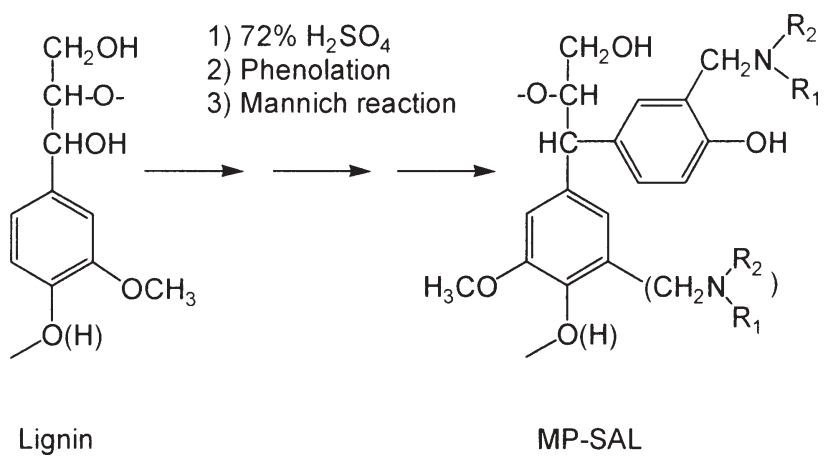

Fig. 1. Preparation of cationic polymer (MP-SAL) from lignin by the Mannich reaction

In this study, the application of MP-SAL prepared from $\mathrm{P}-\mathrm{SAL}$ as retention aids for the rosin emulsion size and soap rosin size under neutral conditions was investigated.

\section{Materials and methods}

\section{Materials}

A commercial bleached hardwood kraft pulp was beaten to $450 \mathrm{ml}$ Canadian Standard Freeness with a TAPPI standard Niagara beater. Rosin emulsion size (Harsize NES-500) and soap rosin size (Harsize L-750) were obtained from Harima. Polyethylenimine (PEI), with a molecular weight of 70000 , was purchased from Tokyo Kasei Kogyo. Cellulose tube (BioDesignDialysis Tubing \#D306-50) for dialysis was obtained from BioDesign.

Preparation of cationic polymer from sulfuric acid lignin

Sulfuric acid lignin (SAL) was prepared from red pine (Pinus densiflora Sieb. et Zucc) by the Klason method. ${ }^{11}$ Phenolation of it was carried out according to our previous procedure. ${ }^{3}$ The cationic polymers were obtained by reaction of P-SAL with amines in the Mannich reaction ${ }^{7}$ at $60^{\circ} \mathrm{C}$ under various conditions as shown in Table 1. After the reaction mixture was dialyzed with a cellulose tube $(3500$ molecular weight cutoff), the reaction products were lyophilized to yield water-soluble cationic polymers (MP-SAL 1-6).

Measurement of nitrogen content and charge density of cationic polymers

Nitrogen content of MP-SAL was measured by elemental analysis using a Perkin Elmer 2400 II elemental analyzer. The charge densities of cationic polymers were determined by colloid titration with a $0.0025 \mathrm{~N}$ solution of potassium polyvinyl sulfate. $^{12}$

\section{Preparation of handsheets}

To a $1.3 \%$ pulp suspension, $0.5 \% \mathrm{NaOH}$ or $0.5 \% \mathrm{H}_{2} \mathrm{SO}_{4}$ solution was added to adjust to the desired $\mathrm{pH}$. Then, the rosin emulsion size or the soap rosin size and MP-SAL were added and stirred for $3 \mathrm{~min}$. In the alum-MPSAL dual system, alum was added before the rosin emulsion size, while the soap rosin size was added before the addition of alum. The $\mathrm{pH}$ of the pulp suspension was measured just before making the handsheet. The pulp suspension was subjected to handsheet making with a basis weight of $60 \mathrm{~g} / \mathrm{m}^{2}$. Wet-pressed handsheets were dried at room temperature for 1 day and then cured at $110^{\circ} \mathrm{C}$ for $30 \mathrm{~min}$ in an oven.

Measurement of Stöckight sizing degree of handsheets

The Stöckight sizing degree of handsheets was measured according to the JIS method. ${ }^{13}$

Measurement of brightness of handsheets

The brightness of handsheets was measured by a Nippon Denshoku ND-300A color difference meter. 


\section{Results and discussion}

Sizing behavior in the MP-SAL single system

Recently, it was reported that some polyamines could serve as retention aids in the rosin size system in neutral or alkaline conditions. ${ }^{14-19}$ In our previous study, ${ }^{9}$ cationic polymers (MP-SAL in Fig. 1) prepared from P-SAL by the Mannich reaction with various amines and formaldehyde exhibited good performance in the rosin-ester emulsion system at neutral $\mathrm{pH}$.

The Mannich reaction mechanism of P-SAL has been confirmed in a separate experiment ${ }^{7}$ with lignin model compounds. The yield of the product ranged from $78 \%$ to $90 \%$. The reaction conditions and the properties of the products are summarized in Table 1. All prepared cationic polymers were soluble in water.

To determine the suitability of MP-SAL as a retention aid, sizing behavior with MP-SAL only (MP-SAL single system) was investigated first. Figure 2 shows the relationship between MP-SAL 1 dosage and the sizing degree of handsheets prepared with MP-SAL 1 and $0.35 \%$ of the rosin emulsion size at $\mathrm{pH}$ 7.5. Addition of $0.2 \%$ MP-SAL 1 did not exhibit sizing effectiveness; however, the sizing degree was raised with the increase of MP-SAL 1 dosage and about 40 s of Stöckight sizing degree was obtained by adding $1 \%$ MP-SAL 1.

To investigate the influence of $\mathrm{pH}$, we prepared handsheets at different $\mathrm{pH}$ levels. The results are shown in Fig. 3. The sizing degrees of the handsheets prepared with rosin emulsion size and MP-SAL 1 were not influenced by $\mathrm{pH}$ and they maintained high effectiveness, whereas the performance of alum was dependent on $\mathrm{pH}$. The handsheets prepared with the soap rosin size and MP-SAL $\mathbf{1}$ did not show any sizing effectiveness at every $\mathrm{pH}$. Wu et



\section{Dosage of MP-SAL (\%)}

Fig. 2. Sizing degrees of handsheets prepared with rosin emulsion size $(0.35 \%)$ and MP-SAL $1(0 \%-1 \%)$ at $\mathrm{pH}$ 7.5. Filled circles, MP-SAL single system; open circles, alum-MP-SAL dual system with $1 \%$ alum al. ${ }^{16}$ also reported that some polyamines were not suitable for soap rosin size under neutral papermaking conditions. This implies that the retention mechanisms of each rosin sizes were different. It was difficult to determine the difference in sizing development mechanism of the rosin emulsion size and soap rosin size in this experiment.

Compared to PEI, a general cationic polymer which possesses a linearly structure, the sizing effectiveness of MPSAL 1, which is considered to be a highly branched polymer because of lignin derivatives, was higher than that of PEI. The cationic charge density of MP-SAL $1(1.3-2 \mathrm{mEq} / \mathrm{g})$ was lower than that of PEI (over $5 \mathrm{mEq} / \mathrm{g}$ ) (Fig. 4). Indeed,

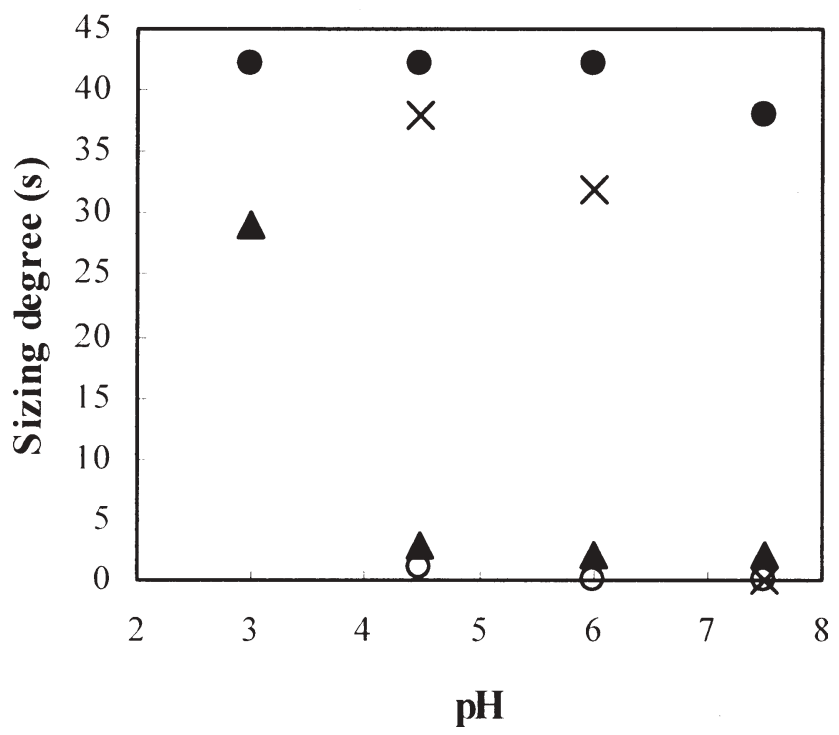

Fig. 3. Effects of sheet-forming $\mathrm{pH}$ on sizing degree of acid rosin sizes $(0.35 \%)$ with cationic polymers $(1 \%)$ or alum $(3 \%)$. Filled circles, rosin emulsion size with MP-SAL 1; filled triangles, rosin emulsion size with polyethylenimine (PEI); crosses, rosin emulsion size with alum; open circles, soap rosin size with MP-SAL 1

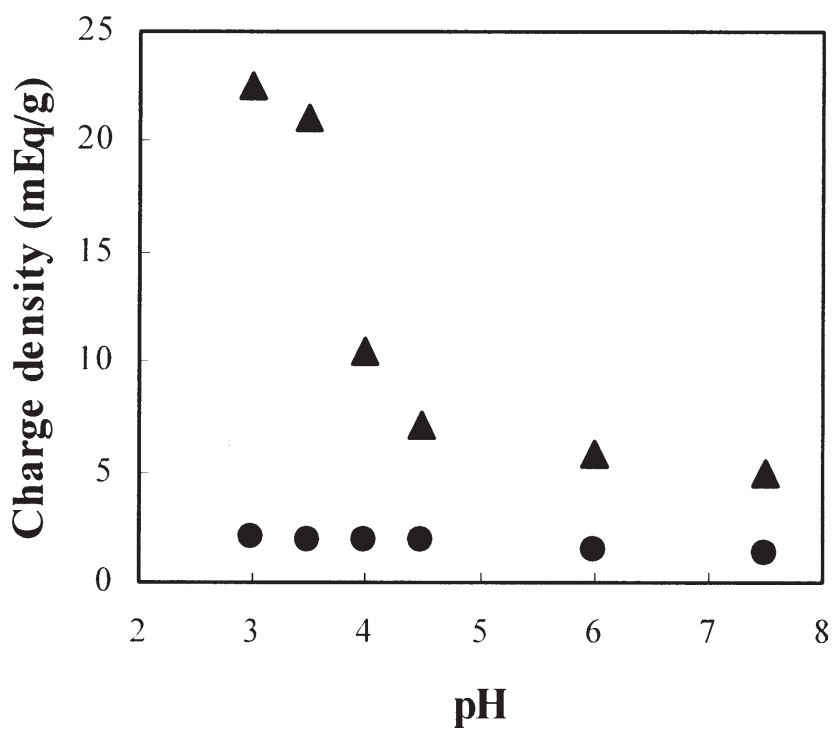

Fig. 4. Effects of $\mathrm{pH}$ on cationic charge density of MP-SAL and PEI. Filled circles, MP-SAL 1; filled triangles, PEI 
Table 2. $\mathrm{p} K_{\mathrm{a}}$ of amines

\begin{tabular}{lr}
\hline & $\mathrm{pKa}$ \\
\hline Trimethylamine & 9.80 \\
Triethylamine & 10.72 \\
Dimethylamine & 10.77 \\
Diethylamine & 10.98 \\
\hline Data reported by Ootaki &
\end{tabular}

Data reported by Ootaki ${ }^{20}$

PEI exhibits a cationic charge density of $22.5 \mathrm{mEq} / \mathrm{g}$ at $\mathrm{pH}$ 3.0. The molecular weights of MP-SAL 1 and PEI were about $10000^{5}$ and 70000 , respectively. These results are in accord with reports ${ }^{14-17,19}$ that the sizing effectiveness was not only affected by charge density and molecular weight to a great extent, but also by the molecular structure of the retention aid.

Table 1 shows the sizing degrees of handsheets prepared with the rosin emulsion size and MP-SALs. Primary and secondary amines as reaction reagents in the Mannich reaction become secondary and tertiary amino groups, respectively, in the MP-SAL. Biermann ${ }^{14}$ concluded that the presence of protonated amines in the polymer chain is important and that the $\mathrm{p} K_{\mathrm{a}}$ value of amines affects the capability of a retention aid. In our previous study, ${ }^{9}$ it was found that MP-SAL with a higher $\mathrm{p} K_{\mathrm{a}}$ value shows higher absorptivity on pulp and exhibits great performance on sizing effectiveness in the rosin-ester emulsion size system. This trend was also observed (Tables 1 and 2) in this study. As expected, the higher amine content of MP-SAL with the same amino group brought an increase in sizing effectiveness (MP-SAL 1-3 in Table 1).

It was found from our results that MP-SAL is effective as a retention aid in neutral papermaking conditions with adequate selection of the rosin.

Sizing behavior in the alum-MP-SAL dual system

Wang and Tanaka ${ }^{19}$ reported that some cationic polymers and alum synergistically enhance the sizing effectiveness in neutral-alkaline papermaking. In this study, MP-SAL was used together with alum (alum-MP-SAL dual system) to investigate the sizing behavior. Figure 2 depicts the sizing degree as a function of MP-SAL dosage with $1 \%$ alum at $\mathrm{pH}$ 7.5. It shows that MP-SAL has a synergistic effect with alum, and more than a $0.05 \%$ dosage of MP-SAL produces high sizing (over $33 \mathrm{~s}$ of Stöckight sizing degree) using the rosin emulsion size. When the soap rosin size was used, sizing effectiveness was not observed. Wang and Tanaka ${ }^{19}$ reported that the polymers with lower charge density were not effective for rosin emulsion size, while the polymers with higher charge density were effective for soap rosin size. No efficiency of MP-SAL in the soap rosin sizing may be attributable to low charge density.

Brightness of handsheets prepared with MP-SAL

The brightness of the handsheets prepared with 1\% MPSAL 1 decreased from $83.8 \%$ to $59.7 \%$. To obtain more
Table 3. Calculation of handsheet brightness by the Kubelka-Munk theory

\begin{tabular}{|c|c|c|}
\hline \multirow{2}{*}{$\frac{\text { Original pulp }}{\text { Brightness (\%) }}$} & \multicolumn{2}{|l|}{ Handsheet } \\
\hline & MP-SAL 1 dosage (\%) & Brightness (\%) \\
\hline \multirow[t]{3}{*}{85} & 1.0 & 60.0 \\
\hline & 0.1 & 79.9 \\
\hline & 0.05 & 82.2 \\
\hline \multirow[t]{3}{*}{50} & 1.0 & 43.4 \\
\hline & 0.1 & 49.2 \\
\hline & 0.05 & 49.6 \\
\hline
\end{tabular}

information regarding the brightness drop, simulation was carried out using the Kubelka-Munk theory ${ }^{9}$ using the MPSAL single system data. These results are shown in Table 3. When the original pulp had $85 \%$ brightness, the brightness decreased to $60.0 \%$ by adding $1 \%$ MP-SAL 1 in the MPSAL single system. In the alum-MP-SAL dual system, however, the dosage of MP-SAL 1 could decrease to $0.05 \%$ to obtain a high sizing degree (Fig. 2) and the brightness decreased only $0.4 \%$ when the original pulp brightness was $50 \%$. Thus, MP-SAL is expected to be usable as a retention aid in newsprinting paper and mechanical papers that do not require a high level of brightness.

\section{Conclusion}

In this study, it was found that the cationic polymers (MPSAL) prepared from sulfuric acid lignin (SAL) by the Mannich reaction possess high potential as retention aids for the rosin emulsion size under neutral papermaking conditions. In the MP-SAL single system, MP-SAL exhibited high sizing effectiveness in neutral papermaking with the rosin emulsion size. In the alum-MP-SAL dual system, alum and MP-SAL synergistically enhanced the sizing effectiveness in the rosin emulsion sizing at neutral $\mathrm{pH}$. To elucidate the sizing development mechanism, further research will be needed.

Acknowledgments The authors thank Harima Chemicals Inc. for providing the acid rosin sizes. This research was conducted with the support of a Grant-in-Aid for Scientific Research (11460079) from the Ministry of Culture, Sports, Science, and Technology of Japan.

\section{References}

1. Yasuda S, Terashima N, Ito T (1981) Chemical structure of sulfuric acid lignin. IV. Reaction of arylglycerol- $\beta$-aryl ether with seventytwo percent sulfuric acid. Mokuzai Gakkaishi 27:879-884

2. Yasuda S, Terashima N (1982) Chemical structure of sulfuric acid lignin. V. Reaction of three arylglycerol- $\beta$-aryl ethers $[\alpha-, \beta$-, and $\gamma$ $\left.{ }^{13} \mathrm{C}\right]$ with seventy-two percent sulfuric acid. Mokuzai Gakkaishi 28:383-387

3. Yasuda S, Tachi M, Takagi Y (1989) Phenolization of red pine sulfuric acid lignin and guaiacyl sulfuric acid lignin model in the presence of sulfuric acid catalyst. Mokuzai Gakkaishi 35:513-520

4. Yasuda S, Ito K, Hamaguchi E, Matsushita Y (1997) Ready chemical conversion of acid hydrolysis lignin into water-soluble ligno- 
sulfonate I. Successive treatment of acid hydrolysis lignin by phenolation, hydroxymethylation and sulfonation. Mokuzai Gakkaishi 43:68-74

5. Yasuda S, Hamaguchi E, Asano K (1999) Ready chemical conversion of acid hydrolysis lignin into water-soluble lignosulfonate. III. Successive treatment of acid hydrolysis lignin and a lignin model compound by phenolation and arylsulfonation. J Wood Sci 45:245249

6. Yasuda S, Asano K (2000) Preparation of strongly acidic cationexchange resins from gymnosperm acid hydrolysis lignin. J Wood Sci 46:477-479

7. Matsushita Y, Yasuda S (2003) Reactivity of a condensed-type lignin model compound in the Mannich reaction and preparation of cationic surfactant from sulfuric acid lignin. J Wood Sci 49:166171

8. Matsushita Y, Yasuda S (2003) Preparation of anion-exchange resins from pine sulfuric acid lignin, one of the acid hydrolysis lignins. J Wood Sci 49:423-429

9. Matsushita Y, Yasuda S (2003) Preparation and evaluation of mordants for neutral rosin size emulsion from lignin. Jpn TAPPI J $57: 882-892$

10. Isogai A (2001) Improvement of surfaces 2. In: Material science of cellulose (in Japanese). University of Tokyo Press, pp 123-130

11. Browning BL (1967) Determination of lignin. In: Methods of Wood Chemistry. Interscience, New York, pp 785-787
12. Tanaka H, Senju R (1976) Hofmann degradation of polyacrylamide (in Japanese). Kobunshi Ronbunshu 33:309-316

13. JIS Method (1979) Testing method for Stöckigt sizing degree of paper. $\mathrm{P} 8122$

14. Biermann CJ (1992) Rosin sizing with polyamine mordants from pH 3 to 10. TAPPI J 75:166-171

15. Wu Z, Tanaka H (1995) Behaviors of polyvinylamines in neutral rosin sizing. Mokuzai Gakkaishi 41:911-916

16. Wu Z, Chen S, Tanaka H (1997) Effects of polyamine structure on rosin sizing under neutral papermaking conditions. J Appl Polym Sci 65:2159-2163

17. Wang F, Tanaka $H$ (2000) Aminated poly- $N$-vinylformamide as a modern retention aid of alkaline paper sizing with acid rosin sizes. J Appl Polym Sci 78:1805-1810

18. Xu Y, Hartong B, Deng Y (2002) Neutral to alkaline rosin sizing using polyethylenimine-epichlorohydrin (PEI-epi) as a mordant. J Pulp Paper Sci 28:39-44

19. Wang F, Tanaka H (2001) Mechanisms of neutral-alkaline paper sizing with usual rosin size using alum-polymer dual retention aid system. J Pulp Paper Sci 27:8-13

20. Ootaki H (1997) Chemical equilibrium. In: Nippon kagakukai (ed), Kagaku binran Kisohen (in Japanese). Maruzen, Tokyo, pp II317-321 